Research Article

\title{
Nasal Carriage of Methicillin-Resistant Staphylococcus aureus among Healthcare Workers in a Tertiary Care Hospital, Kathmandu, Nepal
}

\author{
Nisha Giri $\mathbb{D}^{1},{ }^{1,2}$ Sujina Maharjan $\left(\mathbb{D},{ }^{1}\right.$ Tika Bahadur Thapa ${ }^{(D)},{ }^{1}$ Sushant Pokhrel $\left(\mathbb{D},{ }^{1,3}\right.$ \\ Govardhan Joshi $\mathbb{1}{ }^{1}$ Ojaswee Shrestha $\mathbb{}^{1},{ }^{1}$ Nabina Shrestha $\left(\mathbb{D},{ }^{1}\right.$ \\ and Basista Prasad Rijal (iD ${ }^{4}$ \\ ${ }^{1}$ Department of Laboratory Medicine, Manmohan Memorial Institute of Health Sciences, Soalteemode, Kathmandu, Nepal \\ ${ }^{2}$ Department of Clinical Biochemistry, Dhulikhel Hospital, Kavrepalanchowk, Dhulikhel, Nepal \\ ${ }^{3}$ Department of Genetics Laboratory, National Academy of Medical Sciences, Bir Hospital, Kathmandu, Nepal \\ ${ }^{4}$ Department of Clinical Microbiology, Manmohan Memorial Medical College and Teaching Hospital, Swoyambhu, \\ Kathmandu, Nepal
}

Correspondence should be addressed to Basista Prasad Rijal; basistarijal@gmail.com

Received 19 September 2020; Revised 3 July 2021; Accepted 2 August 2021; Published 11 August 2021

Academic Editor: Simona Nardoni

Copyright (c) 2021 Nisha Giri et al. This is an open access article distributed under the Creative Commons Attribution License, which permits unrestricted use, distribution, and reproduction in any medium, provided the original work is properly cited.

Introduction. Methicillin-resistant Staphylococcus aureus (MRSA) is one of the most common causes of nosocomial infections. One of the potential risk factors for nosocomial staphylococcal infections is colonization of the anterior nares of healthcare workers (HCWs). Our study aimed to determine the rate of nasal carriage MRSA among HCWs at Manmohan Memorial Medical College and Teaching Hospital, Kathmandu. Methods. Two hundred and thirty-two nasal swabs were collected from HCWs of Manmohan Memorial Medical College and Teaching Hospital, Kathmandu, Nepal, within six months (February 2018-July 2018). Nasal swabs were cultured, and $S$. aureus isolates were subjected to the antimicrobial susceptibility test by the modified Kirby-Bauer disc diffusion method. MRSA and iMLSB (inducible macrolide lincosamide streptogramin B) resistance was screened using the cefoxitin disc (30 $\mu \mathrm{g})$ and D-test (clindamycin and erythromycin sensitivity pattern), respectively, following CLSI (Clinical and Laboratory Standard Institute) guidelines. Risk factors for MRSA colonization were determined using the chi-square test considering the $p$ value ${ }^{<} 0.05$ as significant. Results. A total of 34/232 (14.7\%) S. aureus were isolated, out of which 12 (35.3\%) were MRSA. The overall rate of nasal carriage MRSA among HCWs was 5.2\% (12/232). Colonization of MRSA was higher in males (8.7\%) than in females (4.3\%). MRSA colonization was found to be at peak among the doctors (11.4\%). HCWs of the postoperative ward were colonized highest (18.2\%). All MRSA isolates were sensitive to linezolid and tetracycline. iMLSB resistance was shown by $7(20.6 \%)$ of the isolates. MRSA strains showed higher iMLSB resistance accounting for 33.3\% (4/12) in comparison to methicillin-susceptible strains with $13.6 \%(3 / 22)$. Smoking was found to be significantly associated with MRSA colonization $(p=0.004)$. Conclusion. Rate of nasal carriage MRSA is high among HCWs and hence needs special attention to prevent $\mathrm{HCW}$-associated infections that may result due to nasal colonization.

\section{Introduction}

Staphylococcus aureus is a major human pathogen which has potential to cause ample of clinical infections ranging from bacteraemia and infective endocarditis to osteoarticular, skin and soft tissue, pleuropulmonary, and device-related infections [1]. With a few exceptions, the incidence of hospital-acquired infection caused by methicillin-resistant Staphylococcus aureus (MRSA) is increasing worldwide, resulting in longer hospital stay, prolonged antibiotic administration, and higher costs [2].

Penicillin was introduced in the 1940s to treat staphylococcal infections; however, in 1945, strains of S. aureus developed resistance to it. Later, methicillin was introduced in 1959, but in 1961, S. aureus isolates acquired resistance to methicillin as well [3]. MRSA strains exhibit blanket resistance to virtually all $\beta$-lactams, often associated with resistance to other classes of antibiotics [4]. The mecA gene, 
present on the staphylococcal cassette chromosome (SCC), codes for a low antibiotic affinity PBP (penicillin-binding protein) known as $\mathrm{PBP} 2 \mathrm{a}$, responsible for the resistance among MRSA strains [5].

A few options are available for the treatment of MRSAassociated infections, such as macrolides, lincosamides, and streptogramin B (MLSB) with clindamycin being a good alternative, particularly for skin and soft tissue infections, and also works as an alternative in penicillin-allergic patients [6]. However, MLSB resistance is one of the most common resistance mechanisms detected in Gram-positive organism [7]. MLSB resistance can be either constitutive (cMLSB) or inducible (iMLSB) [8]. Failure in detection of iMLSB resistance may be a clinical failure of clindamycin treatment [9]. Since the incidence of inducible clindamycin resistance is high, accurate identification of inducible clindamycin resistance is important to prevent therapeutic failure in infections caused by these strains [10].

It has been reported that HCWs have been the source of MRSA outbreaks in several cases [11]. S. aureus nasal carrier, a nurse, caused outbreaks in two newborn nurseries at different hospitals in association with upper respiratory tract infections in 1986. Phage typing revealed that the nurse's strain of S. aureus and the outbreak strains were identical [12]. Previous studies suggest possibilities that HCWs play a substantial role in MRSA transmission, highlighting the importance of rapid and accurate identification of MRSA carrier HCWs [13]. S. aureus nasal carriage among the general adult population shows global variation [14]. The knowledge of frequency of nasal carriage S. aureus and MRSA among HCWs along with their current antimicrobial profile becomes necessary in the selection of appropriate treatment options for these carriers. Several studies have shown that the elimination of carriage in the anterior nares reduces the incidence of staphylococcal infections [15].

Hence, this study was carried out to determine the rate of nasal carriage $S$. aureus and MRSA along with their antimicrobial profile among HCWs. Also, this study aimed to determine the associated risk factors for nasal carriage MRSA.

\section{Materials and Methods}

2.1. Study Design. A hospital-based cross-sectional study was performed in the Department of Microbiology of Manmohan Memorial Medical College and Teaching Hospital, Kathmandu, Nepal, within six months (February 2018-July 2018) among 238 HCWs with informed written consent. However, six HCWs did not provide consent, and hence, only $232 \mathrm{HCW}$ sere included in the study. All the participants in the study were interviewed with a standard questionnaire for the clinical history and demographic data.

2.2. Inclusion Criteria. All the hospital staff who provided patient care directly were involved in the study.

2.3. Exclusion Criteria. HCWs presented with wound and upper respiratory tract infection in the last three months were excluded from the study.
2.4. Nasal Swab Collection. Nasal swabs were collected using sterile cotton swab moistened with normal saline. The swab was introduced $1-2 \mathrm{~cm}$ in the nasal cavity and rotated 3 times both clockwise and anticlockwise. For each specimen, both nostrils were sampled using the same swab and immediately transported to the laboratory on peptone water. The swabs, after 4 hours of incubation at $37^{\circ} \mathrm{C}$ on peptone water, were inoculated on mannitol salt agar.

2.5. Identification of S. aureus. S. aureus was isolated using mannitol salt agar. The isolates were identified by examination of colony characteristics, Gram staining, oxidase test, catalase test, slide coagulase test, tube coagulase test, and deoxyribonuclease test.

2.6. Detection of MRSA. All isolated S. aureus were tested with $30 \mu \mathrm{g}$ cefoxitin on Muller Hinton Agar (MHA) for MRSA screening. The zone size was interpreted according to CLSI guidelines. An inhibition zone diameter of $\leq 21 \mathrm{~mm}$ was reported as MRSA and $\geq 22 \mathrm{~mm}$ was reported as methicillin-sensitive Staphylococcus aureus (MSSA).

2.7. Antibiotic Susceptibility Testing. Antibiotic susceptibility testing was performed by the modified Kirby-Bauer disc diffusion method on MHA using standard methods as recommended by CLSI guidelines. The antibiotics tested were amikacin $(30 \mu \mathrm{g})$, amoxicillin $(10 \mu \mathrm{g})$, cefepime $(30 \mu \mathrm{g})$, cefotaxime $(30 \mu \mathrm{g})$, cefoxitin $(30 \mu \mathrm{g})$, chloramphenicol $(30 \mu \mathrm{g})$, ciprofloxacin $(5 \mu \mathrm{g})$, clindamycin $(2 \mu \mathrm{g})$, cloxacillin $(10 \mu \mathrm{g})$, cotrimoxazole $(25 \mu \mathrm{g})$, erythromycin $(15 \mu \mathrm{g})$, gentamicin $(10 \mu \mathrm{g})$, linezolid $(30 \mu \mathrm{g})$, ofloxacin $(5 \mu \mathrm{g})$, teicoplanin $(30 \mu \mathrm{g})$, and tetracycline $(30 \mu \mathrm{g})$. The result was interpreted as per the guidelines of CLSI zone size interpretative chart in terms of "sensitive," "resistant," and "intermediate sensitive."

2.8. Detection of $i M L S B$ Resistance. Erythromycin (E) and clindamycin (CD) sensitivity patterns were reported following CLSI guidelines. Isolates were tested for inducible resistance using the D-test. For the detection of iMLSB resistance, $0.5 \mathrm{McF}$ arland equivalent suspension of organism was inoculated into MHA plate as described in the CLSI recommendation. $\mathrm{CD}(2 \mu \mathrm{g})$ and $\mathrm{E}(15 \mu \mathrm{g})$ discs were placed $15 \mathrm{~mm}$ apart from the center on the MHA. Plates were analyzed after 18 hours of incubation at $37^{\circ} \mathrm{C}$. If the $\mathrm{E}$ zone was $\leq 13 \mathrm{~mm}$ and the $\mathrm{CD}$ zone was $\geq 21 \mathrm{~mm}$ and both had a circular shape, the organism was negative for inducible resistance (D-test negative). If the $\mathrm{E}$ zone was $\leq 13 \mathrm{~mm}$ and the $\mathrm{CD}$ zone was $\geq 21 \mathrm{~mm}$ with a $\mathrm{D}$-shaped zone around the $\mathrm{CD}$, the organism was positive for inducible resistance (Dtest positive).

2.9. Data Analysis. Each sample was encoded with an identification number. Similarly, findings were recorded manually and entered into database. The analysis was carried out by SPSS version 20 (IBM Corporation, Armonk, 
NY, USA). Risk factors for MRSA colonization were determined using the chi-square test considering the $p$ value $<0.05$ as significant.

\section{Results}

A total of 232 nasal swabs were collected from HCWs, of which $34(14.7 \%)$ were found to be carriers of $S$. aureus, and among them, 12/34 (35.3\%) HCWs were found to be MRSA carriers. Overall distribution of MRSA was found to be $5.2 \%$ $(12 / 232)$ (Figure 1).

3.1. Demographic Characteristics of the Study Population. The age of study participants ranged from 16 to 65 years, with a mean age of $28.71 \pm 7.8$ years. The age group of $25-35$ years $(47.8 \%)$ participated in the study was in higher frequency followed by $<25$ years (34.1\%), 35-45 years (13.4\%), and least with the age group of $\geq 45$ years $(4.7 \%)$. Females were predominantly in higher fractions $(80.2 \%)$ in the present study (Table 1).

3.2. Distribution of Bacterial Isolates. Distribution of MSSA was the highest in HCWs of the age group 25-35 years with $11.7 \%$ (13/111) and MRSA in the age group $\geq 45$ years with 9.1\% (1/11). MSSA and MRSA were found to be $19.6 \%$ (9/46) and $8.7 \%(4 / 46)$, respectively, in males being higher in comparison to females (Table 1).

Similarly, the prevalence of MSSA was found to be the highest among laboratory staffs with $25 \%$ (5/20), while MRSA was found to be the highest among doctors with $11.4 \%(5 / 44)$ (Table 2).

Distribution of MSSA was found to be highest in laboratory staffs with $19.2 \%(5 / 26)$, while MRSA was found to be the highest in postoperative ward staffs with $18.2 \%(2 / 11)$ (Table 3).

3.3. Antibiotic Susceptibility Pattern of S. aureus. Among 34 isolated $S$. aureus, 12 were methicillin-resistant, whereas 22 were methicillin-sensitive. Beside penicillins and cephalosporins, MRSA strains were lowest sensitive to ofloxacin and ciprofloxacin (25\% each), followed by erythromycin (33.3\%) and gentamicin (50\%). However, all MRSA strains were sensitive to linezolid and tetracycline, highest sensitivity followed by teicoplanin and chloramphenicol ( $91.7 \%$ each), amikacin and clindamycin (83.3\% each), and cotrimoxazole (75\%).

Among MSSA, the lowest sensitivity was found for amoxicillin (36.4\%), followed by erythromycin (40.9\%) and ofloxacin and ciprofloxacin each being equally sensitive (63.6\%). All isolated MSSA strains were found sensitive to cloxacillin, cefotaxime, cefepime, and chloramphenicol in addition to linezolid and tetracycline. MSSA also showed higher sensitivity to amikacin, clindamycin and teicoplanin (95.5\% each), and gentamicin (90.9\%) (Figure 2).

3.4. Erythromycin and Clindamycin Susceptibility Pattern of S. aureus. Among 34 isolates, 12 (35.3\%) were sensitive to both erythromycin and clindamycin and 2 (5.9\%) were

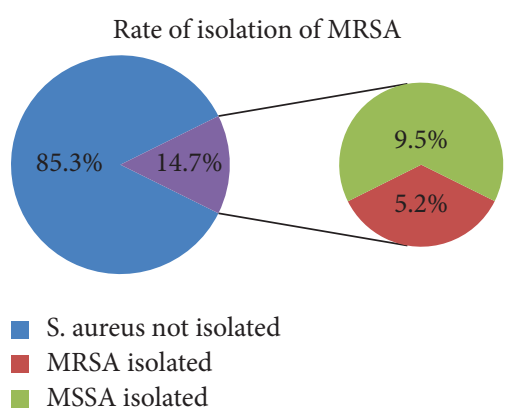

FIGURE 1: Rate of isolation of MRSA.

resistant to both erythromycin and clindamycin. Similarly, $7(20.6 \%)$ were resistant to erythromycin and sensitive to clindamycin with the D-test being positive, whereas 13 (38.2\%) were sensitive to erythromycin and resistant to clindamycin but with the D-test negative. Our study showed higher iMLSB resistance in MRSA strains with $33.3 \%(4 / 12)$ in comparison to MSSA strains with $13.6 \%$ (3/ 22). A total of $7 / 34(20.6 \%)$ of the isolates showed iMLSB resistance (Table 4).

3.5. Determining Risk Factors for MRSA Colonization. Smoking was found to be significantly associated with MRSA colonization $(p=0.004)$. No other factors were significantly associated with MRSA colonization (Table 5).

\section{Discussion}

S. aureus is one of the most common multidrug resistant Gram-positive bacteria and perhaps the pathogen of greatest concern because of its intrinsic virulence, its ability to cause a diverse array of life-threatening infections, and its capacity to adapt to different environmental conditions [16]. In our study, the rate of nasal carriage S. aureus was found to be $14.7 \%$, which is similar to the findings of Khanal et al. (15.7\%) [17] and Khatri et al. (18.3\%) in Nepal [18] and Legese et al. (12.0\%) in Ethiopia [19]. In contrast, our study showed a lower prevalence of nasal carriage $S$. aureus in comparison with the study performed by Vaidya et al. in India (22\%) [20], Chen et al. in China (21.6\%) [21], Boncompain et al. in Argentina (30\%) [22], Moshtagheian et al. in Iran (23.4\%) [23], and several other studies [24-26].

In our study, the overall rate of nasal carriage MRSA was found to be $5.2 \%$. Similar results were found in study performed by Vaidya et al. in India (6\%) [20], Boncompain et al. in Argentina (6.3\%) [22], and other studies [17-19]. However, the prevalence of nasal carriage MRSA in HCWs was found comparatively to be lower in our study in comparison to a study performed in the Gaza strip by Aila et al. (25.5\%) [26]. Whereas, the prevalence of MRSA was found to be slightly higher in our study in comparison to findings of Shrestha et al. in Nepal (2.3\%) [27], Chen et al. in China (1.0\%) [21] and Peters et al. in Germany (1.6\%) [28]. Such differences in the rate of nasal carriage $S$. aureus and MRSA among HCWs may be attributed to different factors 
TABLe 1: Distribution of $S$. aureus by age and sex.

\begin{tabular}{|c|c|c|c|c|c|}
\hline \multirow{3}{*}{ Variable } & \multirow{3}{*}{ Nasal carriage } & \multirow{3}{*}{ Total sample, $n(\%)$} & \multicolumn{3}{|c|}{ S. aureus } \\
\hline & & & \multicolumn{2}{|c|}{ Carriers } & \multirow{2}{*}{ Noncarriers, $n(\%)$} \\
\hline & & & MSSA, $n(\%)$ & MRSA, $n(\%)$ & \\
\hline \multirow{5}{*}{ Age (years) } & $<25$ & $79(34.1)$ & $5(6.3)$ & $4(5.1)$ & $70(88.6)$ \\
\hline & $25-35$ & $111(47.8)$ & $13(11.7)$ & $6(5.4)$ & $92(82.8)$ \\
\hline & $35-45$ & $31(13.4)$ & $3(9.7)$ & $1(3.2)$ & $27(87.1)$ \\
\hline & $\geq 45$ & $11(4.7)$ & $1(9.1)$ & $1(9.1)$ & $9(81.8)$ \\
\hline & Total & $232(100)$ & $22(9.5)$ & $12(5.2)$ & $198(85.3)$ \\
\hline \multirow{3}{*}{ Gender } & Male & $46(19.8)$ & $9(19.6)$ & $4(8.7)$ & $33(71.7)$ \\
\hline & Female & $186(80.2)$ & $13(6.9)$ & $8(4.3)$ & $165(88.7)$ \\
\hline & Total & $232(100)$ & $22(9.5)$ & $12(5.2)$ & $198(85.3)$ \\
\hline
\end{tabular}

Table 2: Profession wise distribution of $S$. aureus.

\begin{tabular}{lcccc}
\hline & & & \multicolumn{2}{c}{ S. aureus } \\
Profession & Total sample, $n(\%)$ & Carriers & Noncarriers, $n(\%)$ \\
\hline Doctor & $44(18.9)$ & $7(15.9)$ & $5(11.4)$ & $32(72.7)$ \\
Laboratory personnel & $20(8.6)$ & $4(25)$ & $0(0)$ & $5(4.7)$ \\
Nurse & $106(45.7)$ & $4(13.8)$ & $1(3.4)$ & $97(91.5)$ \\
Attender & $29(12.5)$ & $1(5)$ & $1(5)$ & $24(82.8)$ \\
Cleaner & $20(8.6)$ & $0(0)$ & $0(0)$ & $18(90.0)$ \\
ANM & $2(0.9)$ & $1(16.7)$ & $0(0)$ & $2(100.0)$ \\
HA & $6(2.6)$ & $0(0)$ & $0(0)$ & $5(83.3)$ \\
CMA & $5(2.2)$ & $22(9.5)$ & $12(5.2)$ & $5(100)$ \\
Total & $232(100)$ & MRSA, $n(\%)$ & $198(85.3)$ \\
\hline
\end{tabular}

ANM, auxiliary nurse midwifery; HA, health assistant; CMA, certified medical assistant.

TABLE 3: Ward wise distribution of S. aureus.

\begin{tabular}{|c|c|c|c|c|}
\hline \multirow{3}{*}{ Ward } & \multirow{3}{*}{ Total sample, $n(\%)$} & \multicolumn{3}{|c|}{ S. aureus } \\
\hline & & \multicolumn{2}{|c|}{ Carriers } & \multirow{2}{*}{ Noncarriers, $n(\%)$} \\
\hline & & MSSA, $n(\%)$ & MRSA, $n(\%)$ & \\
\hline Laboratory & $26(11.2)$ & $5(19.2)$ & $1(3.8)$ & $20(76.9)$ \\
\hline Medical & $24(10.3)$ & $1(4.2)$ & $1(4.2)$ & $22(91.6)$ \\
\hline Cabin & $13(5.6)$ & $1(7.7)$ & $1(7.7)$ & $11(84.6)$ \\
\hline Gynaecology & $14(6.0)$ & $1(7.1)$ & $1(7.1)$ & $12(85.7)$ \\
\hline ICU & $22(9.5)$ & $0(0)$ & $1(4.5)$ & $21(95.5)$ \\
\hline OT & $15(6.5)$ & $2(13.8)$ & $1(6.7)$ & $12(80.0)$ \\
\hline Postoperative & $11(4.7)$ & $2(18.2)$ & $2(18.2)$ & $7(63.6)$ \\
\hline Emergency & $32(13.8)$ & $2(6.3)$ & $1(3.1)$ & $29(90.6)$ \\
\hline Surgical & $27(11.6)$ & $1(3.7)$ & $0(0)$ & $26(96.3)$ \\
\hline OPD & $43(18.5)$ & $7(16.3)$ & $3(6.9)$ & $33(76.7)$ \\
\hline Laundry/CSSD & $5(2.2)$ & $0(0)$ & $0(0)$ & $5(100)$ \\
\hline Total & $232(100)$ & $22(9.5)$ & $12(5.2)$ & $198(85.3)$ \\
\hline
\end{tabular}

ICU, intensive care unit; OT, operation theatre; OPD, outpatient department; CSSD, central sterile services department.

such as sampling techniques, culture methods, identification of $S$. aureus and MRSA basis, study population, study criteria, and hospital environment.

Our study also reported that colonization of MRSA was high in males $(8.7 \%)$ than in females $(4.3 \%)(p>0.05)$. Similarly, Boncompain et al. reported higher MRSA colonization in males $(7.2 \%)$ in comparison to females $(5.8 \%)$ in Argentina [22], while Khatri et al. reported that colonization was higher in females (8.3\%) in comparison to males $(5.1 \%)$ in Nepal [18].
Profession wise, our study showed the highest MRSA colonization of $11.4 \%$ among doctors. Similarly, Vaidya et al. in India reported that doctors were colonized the highest with MRSA [20]. While, Khatri et al. in Nepal reported that lab personnel were the highest nasal carriers of MRSA (10.5\%) [18]. However, the study performed by Haftom et al. in Ethiopia reported MRSA carriage the highest among nurses accounting for 7.8\% [19]. Higher colonization of MRSA among doctors may be due to frequent patient contact. The high rate of nasal carriage MRSA among HCWs indicates high 


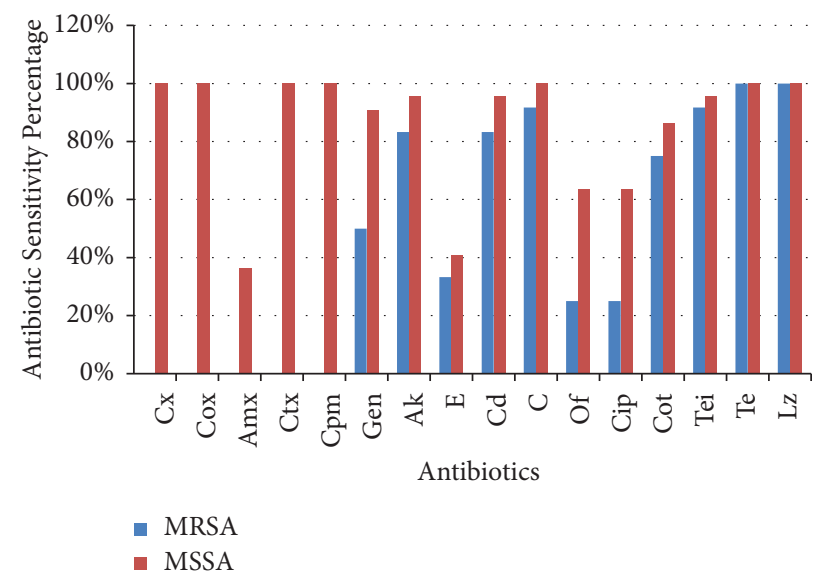

FIgURE 2: Comparison of antibiotic susceptibility pattern between MRSA and MSSA. Cx, cefoxitin; Cox, cloxacillin; Amx, amoxicillin; Ctx, cefotaxime; Cpm, cefepime; Gen, gentamicin; Ak, amikacin; E, erythromycin; Cd, clindamycin; C, chloramphenicol; Of, ofloxacin; Cip, ciprofloxacin; Cot, cotrimoxazole; Tei, teicoplanin; Te, tetracycline; Lz, linezolid.

TABLE 4: Erythromycin and clindamycin susceptibility pattern of S. aureus.

\begin{tabular}{lccc}
\hline $\begin{array}{l}\text { Resistance } \\
\text { pattern }\end{array}$ & S. aureus, $n(\%)$ & MRSA, $n(\%)$ & MSSA, $n(\%)$ \\
\hline E-S, CD-S & $12(35.3)$ & $3(25)$ & $9(40.9)$ \\
E-R, CD-R & $2(5.9)$ & $1(8.4)$ & $1(4.5)$ \\
E-R, CD-S (D+) & $7(20.6)$ & $4(33.3)$ & $3(13.6)$ \\
E-R, CD-S (D-) & $13(38.2)$ & $4(33.3)$ & $9(40.9)$ \\
Total & $34(100)$ & $12(100)$ & $22(100)$ \\
\hline
\end{tabular}

E, erythromycin; CD, clindamycin; $S$, sensitive; $R$, resistance; (D+), D-test positive; (D-), D-test negative.

chances of transmission of the pathogen to the patients during patient care [18], which ultimately leads to longer hospital stay, prolonged antibiotic administration, and higher costs.

Ward wise, our study showed that HCWs of the postoperative ward were colonized the highest (18.2\%). This could be due to the traumatic and postoperative immunological suppression of the patients [29]. As most isolates belonged to HCWs from the postoperative ward, the vulnerability of surgical site wound infection with MRSA among patients, following transmission from carrier HCWs, cannot be ignored. Similarly, Khatri et al. in Nepal reported that the percentages of nasal carriage MRSA (14.3\%) were the highest among HCWs from postoperative department [18]. However, Vaidya et al. in India reported that HCWs of the emergency ward were found to be colonized the highest (15.3\%) [20]. In contrast, a study performed by Haftom et al. in Ethiopia reported that HCWs of the surgical ward were the highest colonized with MRSA (17.1\%) [19].

In our study, all isolated $S$. aureus (both MRSA and MSSA) were sensitive to linezolid and tetracycline. However, in a study performed by Karimi et al., only $58.4 \%$ of S. aureus was sensitive to tetracycline [30]. MRSA strains also showed higher sensitivity to teicoplanin $(91.7 \%)$, chloramphenicol (91.7\%), amikacin (83.3\%), and clindamycin (83.3\%) in our study. High sensitivity of those antibiotics towards MRSA indicates that these antibiotics might be an option for empirical therapy of MRSA infections in our hospital. Similarly, all isolated MSSA were sensitive to chloramphenicol besides cloxacillin, cefoxitin, cefotaxime, and cefepime, followed by teicoplanin (95.5\%), amikacin (95.5\%), clindamycin (95.5\%), gentamicin (90.9\%), and cotrimoxazole $(86.4 \%)$. Similarly, the abovementioned antibiotics might be implicated for the empirical therapy of MSSA infections in our hospital.

Among 34 isolates, 7 (20.6\%) were resistant to erythromycin and sensitive to clindamycin with the D-test being positive. D-test positive was higher (33.3\%) in MRSA in comparison to MSSA (13.6\%). Our study showed lower iMLSB resistance (33.3\%) among MRSA strains in comparison to a study performed by Khanal et al. in Nepal (66.7\%) [17]. The differences in findings may be due to various factors such as different study populations, different study periods, and different settings and variations in methodology. Whereas, in a study performed by Adhikari et al. in Nepal, in clinical samples, iMLSB resistance and CMLSB resistance were found to be $11.48 \%$ and $29.25 \%$, respectively. Comparatively, iMLSB resistance was found to be higher and CMLSB lower in our study [31]. The differences in findings may be due to differences in samples (nasal swabs vs. clinical samples).

Our study also aimed to determine the risk factors for MRSA colonization. The colonization of MRSA was significantly high among smokers $(p=0.004)$. Cigarette smoking increases mucus production, impairs epithelial elastic properties, decreases IgA production, and affects phagocyte activities which facilitate bacterial colonization and exacerbate inflammatory responses leading to epithelial damage, further impairing host immunity and promoting bacterial colonization of the respiratory tract [32]. Coinciding with our study, smoking was found to be one of the risk factors for colonization among healthy adults [33].

In our study, other factors were not found to be significantly associated with MRSA colonization. However, years of service and level of education were found to be significantly associated with MRSA colonization in a study performed by Maroof et al. in India [34]. 
TABle 5: Analysis of potential risk factors associated with MRSA colonization.

\begin{tabular}{|c|c|c|c|c|c|}
\hline Variable & Categories & Total sample $(n=232)$ & MSSA $(n=22)$ & MRSA $(n=12)$ & $P$ value \\
\hline \multirow{4}{*}{ Age (years) } & $<25$ & 79 & $5(6.3 \%)$ & $4(5.1 \%)$ & \multirow{4}{*}{0.845} \\
\hline & $25-35$ & 111 & $13(11.7 \%)$ & $6(5.4 \%)$ & \\
\hline & $35-45$ & 31 & $3(9.7 \%)$ & $1(3.2 \%)$ & \\
\hline & $\geq 45$ & 11 & $1(9.1 \%)$ & $1(9.1 \%)$ & \\
\hline \multirow{2}{*}{ Sex } & Male & 46 & $9(19.6 \%)$ & $4(8.7 \%)$ & \multirow{2}{*}{0.664} \\
\hline & Female & 186 & $13(6.9 \%)$ & $8(4.3 \%)$ & \\
\hline \multirow{11}{*}{ Associated ward } & Laboratory & 26 & $5(19.2 \%)$ & $1(3.8 \%)$ & \multirow{11}{*}{0.885} \\
\hline & Medical & 24 & $1(4.2 \%)$ & $1(4.2 \%)$ & \\
\hline & Cabin & 13 & $1(7.7 \%)$ & $1(7.7 \%)$ & \\
\hline & Gynaecology & 14 & $1(7.1 \%)$ & $1(7.1 \%)$ & \\
\hline & ICU & 22 & 0 & $1(4.5 \%)$ & \\
\hline & OT & 15 & $2(13.8 \%)$ & $1(6.7 \%)$ & \\
\hline & Postoperative & 11 & $2(18.2 \%)$ & $2(18.2 \%)$ & \\
\hline & Emergency & 32 & $2(6.3 \%)$ & $1(3.1 \%)$ & \\
\hline & Surgical & 27 & $1(3.7 \%)$ & 0 & \\
\hline & OPD & 43 & $7(16.3 \%)$ & $3(6.9 \%)$ & \\
\hline & Laundry/CSSD & 5 & 0 & 0 & \\
\hline \multirow{8}{*}{ Occupation } & Doctor & 44 & $7(15.9 \%)$ & $5(11.4 \%)$ & \multirow{8}{*}{0.326} \\
\hline & Lab technicians & 20 & $5(25 \%)$ & 0 & \\
\hline & Nurse & 106 & $4(3.8 \%)$ & $5(4.7 \%)$ & \\
\hline & Attender & 29 & $4(13.8 \%)$ & $1(3.4 \%)$ & \\
\hline & Cleaner & 20 & $1(5.1 \%)$ & $1(5.0 \%)$ & \\
\hline & ANM & 2 & 0 & 0 & \\
\hline & HA & 6 & $1(16.7 \%)$ & 0 & \\
\hline & CMA & 5 & 0 & 0 & \\
\hline \multirow{5}{*}{ Years of service (years) } & $<1$ & 65 & $2(4.5 \%)$ & $4(9.1 \%)$ & \multirow{5}{*}{0.523} \\
\hline & $1-5$ & 113 & $8(7.5 \%)$ & $3(2.8 \%)$ & \\
\hline & $6-10$ & 31 & $7(13.2 \%)$ & $3(5.7 \%)$ & \\
\hline & $11-15$ & 13 & $3(20 \%)$ & $1(6.7 \%)$ & \\
\hline & $>15$ & 10 & $2(14.3 \%)$ & $1(7.1 \%)$ & \\
\hline \multirow{2}{*}{ History of recurrent URTI ( 3 months before) } & Yes & 63 & $6(9.5 \%)$ & $6(9.5 \%)$ & \multirow{2}{*}{0.185} \\
\hline & No & 169 & $16(9.5 \%)$ & $6(3.6 \%)$ & \\
\hline \multirow{2}{*}{ Nasal medication } & Yes & 14 & $1(7.14 \%)$ & 0 & \multirow{2}{*}{0.453} \\
\hline & No & 218 & $21(9.6 \%)$ & $12(5.5 \%)$ & \\
\hline \multirow{2}{*}{ Nasal abnormalities } & Yes & 11 & $1(9.1 \%)$ & 0 & \multirow{2}{*}{0.453} \\
\hline & No & 221 & $21(9.5 \%)$ & $12(5.4 \%)$ & \\
\hline \multirow{2}{*}{ Patient contact } & Yes & 185 & $16(8.6 \%)$ & $11(5.9 \%)$ & \multirow{2}{*}{0.192} \\
\hline & No & 47 & $6(12.8 \%)$ & $1(2.1 \%)$ & \\
\hline \multirow{2}{*}{ Infection control training } & Yes & 7 & $1(14.3 \%)$ & $1(14.3 \%)$ & \multirow{2}{*}{0.645} \\
\hline & No & 225 & $21(9.3 \%)$ & $11(4.9 \%)$ & \\
\hline \multirow{4}{*}{ Level of education } & Illiterate & 15 & $1(6.7 \%)$ & $1(6.7 \%)$ & \\
\hline & Undergraduate & 141 & $12(8.5 \%)$ & $5(3.5 \%)$ & \\
\hline & Graduate & 73 & $9(12.3 \%)$ & $6(8.2 \%)$ & 0.744 \\
\hline & Postgraduate & 3 & 0 & 0 & \\
\hline & Yes & 80 & $6(7.5 \%)$ & $3(3.8 \%)$ & \\
\hline History of hospitalization for $>24 \mathrm{~h}$ & No & 152 & $16(10.5 \%)$ & $9(5.9 \%)$ & 0.886 \\
\hline & Yes & 38 & $5(13.2 \%)$ & $1(2.6 \%)$ & \\
\hline Intake of antibiotics within 3 months & No & 194 & $17(8.8 \%)$ & $11(5.7 \%)$ & 0.293 \\
\hline Smoking habits & Yes & 11 & 0 & $4(36.4 \%)$ & 0.004 \\
\hline Smoking habits & No & 221 & $22(9.9 \%)$ & $8(3.6 \%)$ & 0.004 \\
\hline
\end{tabular}

MSSA, methicillin-sensitive Staphylococcus aureus; MRSA, methicillin-resistant Staphylococcus aureus; OR, odds ratio; CI, confidence interval; ICU, intensive care unit; OT, operation theatre; ER, emergency; OPD, outpatient department; CSSD, central sterile services department; ANM, auxiliary nurse midwifery; HA, health assistant; CMA, certified medical assistant; URTI, upper respiratory tract infectionBold face indicates $p$ value $<0.05$.

Regular surveillance and decolonization of MRSApositive HCWs can help as an effective measure to control MRSA infection. Similarly, an awareness campaign in the HCWs may be reinforced to take adequate care and precautions regarding the universal techniques of hand washing and hygiene thereby minimizing risks of transmitting hospital-acquired MRSA. Furthermore, the association between carriage and infections can be established by 
identifying strains with the same genotype that helps to add further evidence on the relationship between nasal carriage MRSA among HCWs and incidence of staphylococcal infections among patients.

This study is based only on the phenotypic methods. It reveals only about the carriage status of $S$. aureus (carriage status of other organisms such as coagulase-negative S. aureus was not studied). Also, this study only determines the carriage status but does not suggest about the ways for its decolonization.

\section{Conclusion}

Our study revealed that the nasal carriage $S$. aureus and MRSA is high among HCWs, especially among the doctors and the HCWs of the postoperative ward. iMLSB resistance is higher in MRSA than in MSSA strains. The high rate of nasal carriage MRSA among HCWs indicates the need for standard infection control precautions to be employed in the professional practice to minimize the carriage as well as the transmission rate. Our study also suggested that smoking is significantly associated with MRSA colonization.

\section{Data Availability}

The data generated to support the findings of this study are included within the article. The primary raw data are available to interested researchers from the corresponding author upon request.

\section{Ethical Approval}

Ethical approval was taken from the Institutional Research Council (IRC) of Manmohan Memorial Institute of Health Sciences (MMIHS): MMIHS-IRC 224 and Department of Microbiology, Manmohan Memorial Medical College and Teaching Hospital, Kathmandu, Nepal.

\section{Consent}

Written informed consent was taken from all the participants involved in the study.

\section{Conflicts of Interest}

The authors declare that there are no conflicts of interest.

\section{Acknowledgments}

The authors would like to share their deepest gratitude to the Department of Clinical Microbiology and all the HCWs of Manmohan Memorial Medical College and Teaching Hospital and the faculty of the Laboratory Medicine of Manmohan Memorial Institute of Health Sciences for their support and International Journal of Infectious Disease for recognizing their previous work (https://www.ijidonline. com/article/S1201-9712(20)31446-6/pdf).

\section{References}

[1] S. Y. C. Tong, J. S. Davis, E. Eichenberger, T. L. Holland, and V. G. Fowler Jr., "Staphylococcus aureus infections: epidemiology, pathophysiology, clinical manifestations, and management," Clinical Microbiology Reviews, vol. 28, no. 3, pp. 603-661, 2015.

[2] P. Kumar, I. Shukla, and S. Varshney, "Nasal screening of healthcare workers for nasal carriage of coagulase positive MRSA and prevalence of nasal colonization with Staphylococcus aureus," Biology and Medicine, vol. 3, pp. 182-186, 2011.

[3] M. Askarian, A. Zeinalzadeh, A. Japoni, A. Alborzi, and Z. A. Memish, "Prevalence of nasal carriage of methicillinresistant Staphylococcus aureus and its antibiotic susceptibility pattern in healthcare workers at Namazi Hospital, Shiraz, Iran," International Journal of Infectious Diseases, vol. 13, no. 5, pp. e241-e247, 2009.

[4] A. Zapun, C. Contreras-Martel, and T. Vernet, "Penicillinbinding proteins and $\beta$-lactam resistance," FEMS Microbiology Reviews, vol. 32, no. 2, pp. 361-385, 2008.

[5] T. Coates, R. Bax, and A. Coates, "Nasal decolonization of Staphylococcus aureus with mupirocin: strengths, weaknesses and future prospects," Journal of Antimicrobial Chemother$a p y$, vol. 64, no. 1, pp. 9-15, 2009.

[6] A. Abbas, P. Srivastava, and P. S. Nirwan, "Prevalence of MLSB resistance and observation of erm A \& erm C genes at A tertiary care hospital," Journal of Clinical and Diagnostic Research: Journal of Clinical and Diagnostic Research, vol. 9, no. 6, pp. DC08-DC10, 2015.

[7] B. Kaskatepe, "Determination of inducible clindamycin resistance in staphylococci strains isolated from clinical samples," Turkish Journal of Pharmaceutical Sciences, vol. 11, no. 3, 2014.

[8] P. C. Schreckenberger, E. Ilendo, and K. L. Ristow, "Incidence of constitutive and inducible clindamycin resistance in Staphylococcus aureus and coagulase-negative staphylococci in a community and a tertiary care hospital," Journal of Clinical Microbiology, vol. 42, no. 6, pp. 2777-2779, 2004.

[9] M. Motamedifar, H. S. E. Sarai, and D. Mansury, "Patterns of constitutive and inducible clindamycin resistance in Staphylococcus aureus isolated from clinical samples by $\mathrm{D}$-test method, shiraz, southwest of Iran," Galen Medical Journal, vol. 3, no. 4, pp. 216-221, 2014

[10] G. S. Ajantha, R. D. Kulkarni, J. Shetty, C. Shubhada, and P. Jain, "Phenotypic detection of inducible clindamycin resistance among Staphylococcus aureus isolates by using the lower limit of recommended inter-disk distance," Indian Journal of Pathology \& Microbiology, vol. 51, no. 3, pp. 376378, 2008.

[11] R. Sassmannshausen, R. H. Deurenberg, R. Köck et al., "MRSA prevalence and associated risk factors among healthcare workers in non-outbreak situations in the Dutch-German EUREGIO," Frontiers in Microbiology, vol. 7, no. 1273, 2016.

[12] R. Sherertz, S. Bassetti, and B. Bassetti-Wyss, “"Cloud” healthcare workers," Emerging Infectious Diseases, vol. 7, no. 2, pp. 241-244, 2001.

[13] M. H. Kim, W. I. Lee, and S. Y. Kang, "Detection of methicillin-Resistant Staphylococcus aureusin healthcare workers using real-time polymerase chain reaction," Yonsei Medical Journal, vol. 54, no. 5, pp. 1282-1284, 2013.

[14] J. U. Sollid, A. S. Furberg, A. M. Hanssen, and M. Johannessen, "Staphylococcus aureus: determinants of 
human carriage," Infection, Genetics and Evolution: Journal of Molecular Epidemiology and Evolutionary Genetics in Infectious Diseases, vol. 21, pp. 531-541, 2014.

[15] N. Akhtar, "Staphylococcal nasal carriage of health care workers," Journal of the College of Physicians and SurgeonsPakistan: JCPSP, vol. 20, no. 7, pp. 439-443, 2010.

[16] F. D. Lowy, "Antimicrobial resistance: the example of Staphylococcus aureus," Journal of Clinical Investigation, vol. 111, no. 9, pp. 1265-1273, 2003.

[17] R. Khanal, P. Sah, P. Lamichhane, A. Lamsal, S. Upadhaya, and V. K. Pahwa, "Nasal carriage of methicillin resistant Staphylococcus aureus among health care workers at a tertiary care hospital in Western Nepal," Antimicrobial Resistance and Infection Control, vol. 4, no. 1, p. 39, 2015.

[18] S. Khatri, N. D. Pant, R. Bhandari et al., "Nasal carriage rate of methicillin resistant Staphylococcus aureus among health care workers at a tertiary care hospital in Kathmandu, Nepal," Journal of Nepal Health Research Council, vol. 15, no. 35, pp. 26-30, 2017.

[19] H. Legese, A. G. Kahsay, A. Kahsay et al., "Nasal carriage, risk factors and antimicrobial susceptibility pattern of methicillin resistant Staphylococcus aureus among healthcare workers in Adigrat and Wukro hospitals, Tigray, Northern Ethiopia," BMC Research Notes, vol. 11, no. 1, p. 250, 2018.

[20] V. Rutvi, D. P. Sangeeta, K. B. Sima, and A. P. Piyush, "Nasal carriage rate of methicillin resistant Staphylococcus aureus (MRSA) among civil hospital health care workers," International Journal of Medicine and Public Health, vol. 6, no. 4, pp. 180-183, 2016.

[21] B. Chen, X. Dai, B. He et al., "Differences in Staphylococcus aureus nasal carriage and molecular characteristics among community residents and healthcare workers at Sun Yat-Sen University, Guangzhou, Southern China," BMC Infectious Diseases, vol. 15, no. 1, p. 303, 2015.

[22] C. A. Boncompain, C. A. Suárez, and H. R. Morbidoni, "Staphylococcus aureus nasal carriage in health care workers: first report from a major public hospital in Argentina," Revista Argentina de Microbiología, vol. 49, no. 2, pp. 125-131, 2017.

[23] S. Moshtagheian, M. Halaji, H. Sedaghat et al., "Molecular characteristics of methicillin-resistant Staphylococcus aureus nasal carriage from hospitalized patients and medical staff in Isfahan, Iran," Annali di Igiene: Medicina Preventiva e di Comunita, vol. 30, no. 3, pp. 237-244, 2018.

[24] A. Shibabaw, T. Abebe, and A. Mihret, "Nasal carriage rate of methicillin resistant Staphylococcus aureus among dessie referral hospital health care workers; dessie, northeast Ethiopia," Antimicrobial Resistance and Infection Control, vol. 2, no. 1, p. 25, 2013.

[25] S. R. Rongpharpi, N. K. Hazarika, and H. Kalita, "The prevalence of nasal carriage of Staphylococcus aureus among healthcare workers at a tertiary care hospital in Assam with special reference to MRSA," Journal of Clinical and Diagnostic Research: Journal of Clinical and Diagnostic Research, vol. 7, no. 2, pp. 257-60, 2013.

[26] N. A. El Aila, N. A. Al Laham, and B. M. Ayesh, "Nasal carriage of methicillin resistant Staphylococcus aureus among health care workers at Al Shifa hospital in Gaza Strip," BMC Infectious Diseases, vol. 17, no. 1, p. 28, 2017.

[27] B. Shrestha, B. M. Pokhrel, and T. M. Mohapatra, "Staphylococcus aureus nasal carriage among health care workers in a Nepal Hospital," Brazilian Journal of Infectious Diseases, vol. 13, no. 5, p. 322, 2009.

[28] C. Peters, M. Dulon, O. Kleinmüller, A. Nienhaus, and A. Schablon, "MRSA prevalence and risk factors among health personnel and residents in nursing homes in hamburg, Germany-a cross-sectional study," PloS One, vol. 12, no. 1, Article ID e0169425, 2017.

[29] H. Al-Talib, C. Y. Yean, H. Hasan, N. Nik Zuraina, and M. Ravichandran, "Methicillin-resistant Staphylococcus aureus nasal carriageamong patients and healthcare workers in a hospital in kelantan, Malaysia," Polish Journal of Microbiology, vol. 62, no. 1, pp. 109-112, 2013.

[30] M. Karimi, B. N. Esfahani, M. Halaji et al., "Molecular characteristics and antibiotic resistance pattern of Staphylococcus aureus nasal carriage in tertiary care hospitals of Isfahan, Iran," Infezioni in Medicina, vol. 25, no. 3, pp. 234240, 2017.

[31] R. P. Adhikari, S. Shrestha, A. Barakoti, and R. Amatya, "Inducible clindamycin and methicillin resistant Staphylococcus aureus in a tertiary care hospital, Kathmandu, Nepal," BMC Infectious Diseases, vol. 17, no. 1, p. 483, 2017.

[32] E. K. McEachern, J. H. Hwang, K. M. Sladewski et al., "Analysis of the effects of cigarette smoke on staphylococcal virulence phenotypes," Infection and Immunity, vol. 83, no. 6, pp. 2443-2452, 2015.

[33] C. S. Choi, C. S. Yin, A. A. Bakar et al., "Nasal carriage of Staphylococcus aureus among healthy adults," Journal of Microbiology, Immunology, and Infection, vol. 39, no. 6, pp. 458-464, 2006.

[34] P. Maroof, R. Nasir, N. Bali, A. Farhana, M. Amin, and F. Kanth, "Staphylococcus aureus: nasal-carriage in health care workers and in-patients with special reference to MRSA," British Microbiology Research Journal, vol. 13, no. 4, pp. 1-8, 2016. 